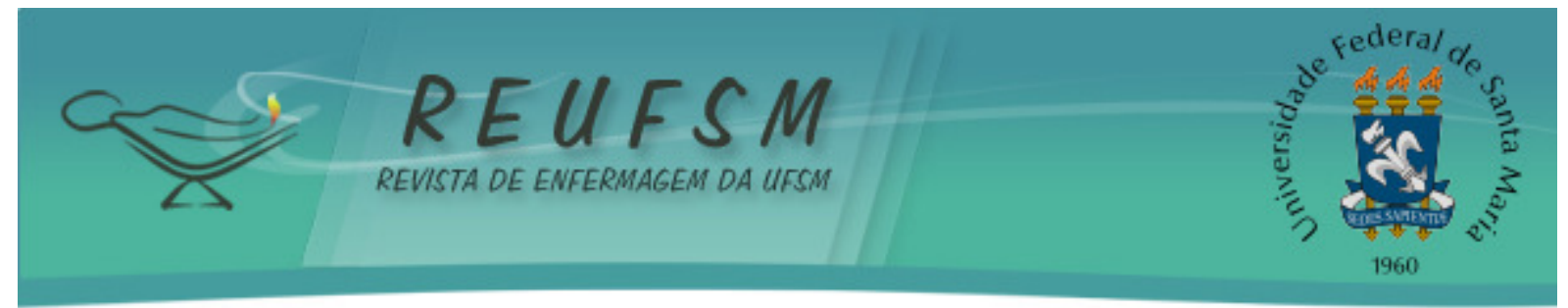

ARTIGO ORIGINAL

\title{
REGISTRO DA AVALIAÇÃO DE ENFERMAGEM EM TERAPIA INTENSIVA: DISCURSO DO SUJEITO COLETIVO
}

\author{
REGISTER OF NURSING EVALUACION IN INTENSIVE CARE:COLLECTIVE SUBJECT'S \\ SPEECH
}

\section{REGISTRO DE EVALUACIÓN DE ENFERMERÍA EN CUIDADOS INTENSIVOS: DISCURSO DEL SUJETO COLECTIVO}

\author{
Aline Colaço ${ }^{1}$ \\ Fernanda Rosado Menezes ${ }^{2}$ \\ Eliane Regina Pereira do Nascimento ${ }^{3}$ \\ Daniele Delacanal Lazzari ${ }^{4}$ \\ Adilson Adair Böes ${ }^{5}$ \\ Walnice Jung ${ }^{6}$
}

Doi: $10.5902 / 2179769215509$

RESUMO: Objetivo: compreender as percepções de enfermeiros sobre a avaliação de enfermagem e seu registro em uma Unidade de Terapia Intensiva. Método: trata-se de um estudo descritivo de caráter qualitativo, desenvolvido com nove enfermeiros de um hospital público, situado em um município do sul do Brasil. Os dados foram coletados mediante roteiro de entrevista semiestruturado e a análise realizada por meio do Discurso do Sujeito Coletivo. Resultados: apontaram a utilização de apenas partes do processo de enfermagem, suas potencialidades e fragilidades. A praticidade e a rapidez para a execução dos registros são aspectos considerados positivos e a insuficiência de outros critérios de avaliação e a superficialidade das informações, como negativos. Considerações Finais: a utilização incompleta do Processo de Enfermagem permanece uma dificuldade para enfermeiros. Há necessidade de propostas aplicáveis que permitam aos enfermeiros novas abordagens na prática, contribuindo para qualificar os registros, favorecendo a segurança o paciente.

Descritores: Registros de enfermagem; Unidade de terapia intensiva; Avaliação em saúde; Avaliação de processos.

ABSTRACT: Aim: to understand the perceptions of nurses about the records of the nursing process in a intensive care unit. Method: descriptive qualitative study, developed with nine nurses in a public hospital located in a county in southern Brazil. Data were collected through semi-structured interview script and the analysis was performed through the Collective Subject Discourse. Results: they showed the use of only parts of the nursing process, its strengths and weaknesses. The practicality and quickness to implement the records are considered positive aspects and the failure of other evaluation criteria, and

\footnotetext{
${ }^{1}$ Enfermeira. Especialista em Enfermagem em Alta Complexidade. Mestranda em Enfermagem na Universidade Federal de Santa Catarina. Florianópolis, Santa Catarina, Brasil. Email: aline.colaco216@gmail.com

${ }^{2}$ Enfermeira. Especialista em Terapia Intensiva. Enfermeira do Hospital Universitário Polydoro E Ernani de São Thiago. Florianópolis, Santa Catarina, Brasil. Email: femenezes@hotmail.com

${ }^{3}$ Enfermeira. Doutora em Enfermagem. Docente da Universidade Federal de Santa Catarina. Florianópolis, Santa Catarina, Brasil. Email: eliane.nascimento@ufsc.br

${ }^{4}$ Enfermeira. Mestre em Educação. Doutoranda em Enfermagem. Universidade Federal de Santa Catarina. Bolsista CNPq. Florianópolis, Santa Catarina, Brasil. Email: danielelazza@gmail.com

${ }^{5}$ Enfermeiro. Mestre em Biologia Aplicada à Saúde. Docente da Universidade Feevale. Novo Hamburgo, Rio Grande do Sul, Brasil. Email: aab@feevale.br

${ }^{6}$ Enfermeira. Especialista em Educação. Mestranda em Enfermagem na Universidade Federal de Santa Catarina. Bolsista CAPES. Florianópolis, Santa Catarina, Brasil. Email: walnicejung@gmail.com
} 


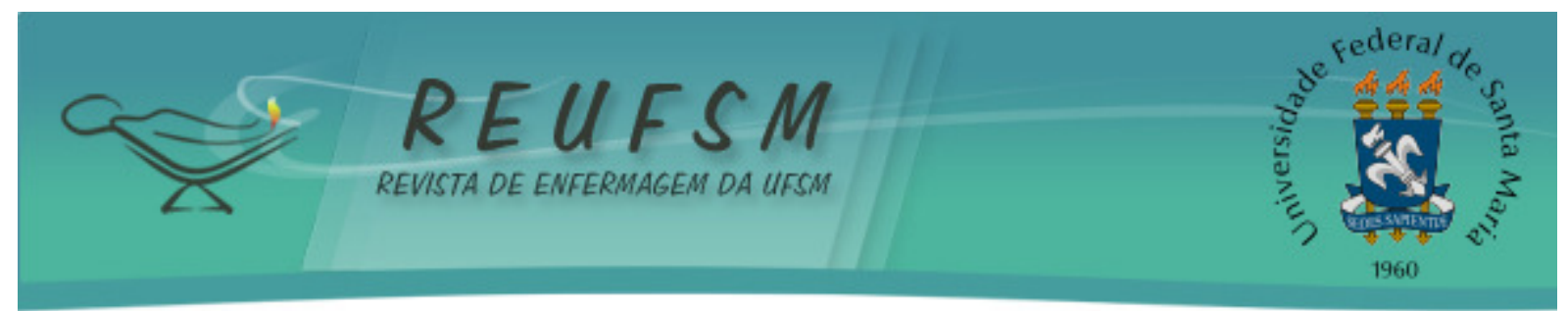

the shallowness of information, such as negative. Final remarks: incomplete utilization of nursing process remains a difficulty for nurses. There is need for proposals that allow nurses to apply new approaches in practice, helping to qualify the records, promoting patient safety.

Descriptors: Nursing records; Intensive care unit; Health evaluation; Process assessment.

RESUMEN: Objetivo: conocer las percepciones de las enfermeras sobre el proceso de evaluación de enfermería y su registro en una Unidad de Cuidados Intensivos. Método: estudio cualitativo descriptivo, desarrollado con nueve enfermeras de un hospital público en Brasil. Los datos fueron recolectados a través de entrevista y el análisis realizado por medio de la técnica del Discurso del Sujeto Colectivo. Resultados: demostraron el uso de sólo algunas partes del proceso de enfermería, sus potencialidades y debilidades. La practicidad y rapidez para poner en práctica los documentos son los aspectos positivos y el fracaso de los otros criterios de evaluación y la poca profundidad de la información, negativos. Consideraciones finales: el uso incompleto del Proceso de Enfermería sigue siendo una dificultad para las enfermeras. Existe la necesidad de propuestas que permitan a las enfermeras nuevos enfoques en la práctica, ayudando a calificar los registros y promoviendo de la seguridad del paciente.

Descriptores: Registros de enfermería; Unidad de cuidados intensivos; Evaluación en salud; Evaluacion de proceso.

\section{INTRODUÇÃO}

0 cuidado de enfermagem ofertado a pacientes em terapia intensiva envolve a tomada de decisões que implicam em questões éticas, opções terapêuticas e demandas gerenciais. $\mathrm{Na}$ avaliação clínica, as evidências informam se as condutas são eficazes e auxiliam na utilização das melhores práticas: o cuidado certo, no momento certo, da maneira certa, para o paciente certo, objetivando alcançar os melhores resultados possíveis. ${ }^{1}$ A partir disso, o julgamento clínico auxilia na análise situacional para definição das ações a serem adotadas para determinados pacientes.

Para o julgamento clínico, a Enfermagem se alicerça em modelos da prática, tais como o Processo de Enfermagem (PE). O PE é o arranjo das ações de Enfermagem sistematizadas e inter-relacionadas com o objetivo de produzir uma assistência de qualidade, por meio da identificação de situações de saúde-doença e necessidades de cuidados. Subsidiam, portanto, planos de cuidados e intervenções específicas para a promoção e recuperação da saúde. ${ }^{2}$

Destaca-se ainda, como fator de importância do $\mathrm{PE}$, a possibilidade de comunicação permanente entre os profissionais da equipe de saúde, além de servir como meio de proteção legal e administrativa para o enfermeiro, favorecendo a visibilidade do trabalho da equipe de Enfermagem, bem como a construção de uma base de dados para pesquisas científicas na área. ${ }^{3}$

O PE se estrutura em cinco etapas inter-relacionadas e recorrentes: coleta de dados, diagnóstico de enfermagem, planejamento, implementação e avaliação. ${ }^{3}$ Essas fases têm por finalidade identificar as necessidades do indivíduo, planejar estratégias de atuação, traçar os objetivos, intervir sobre uma dada situação e avaliar os efeitos desse processo. Essa estrutura promove, portanto, um cuidado orientado para a obtenção de resultados. $^{4}$

A avaliação de enfermagem, didaticamente, se constitui na última etapa do PE e é definida como um método deliberativo, sistemático e ininterrupto de análise das mudanças nas respostas humanas. Por meio da avaliação de enfermagem, é possível determinar se as 


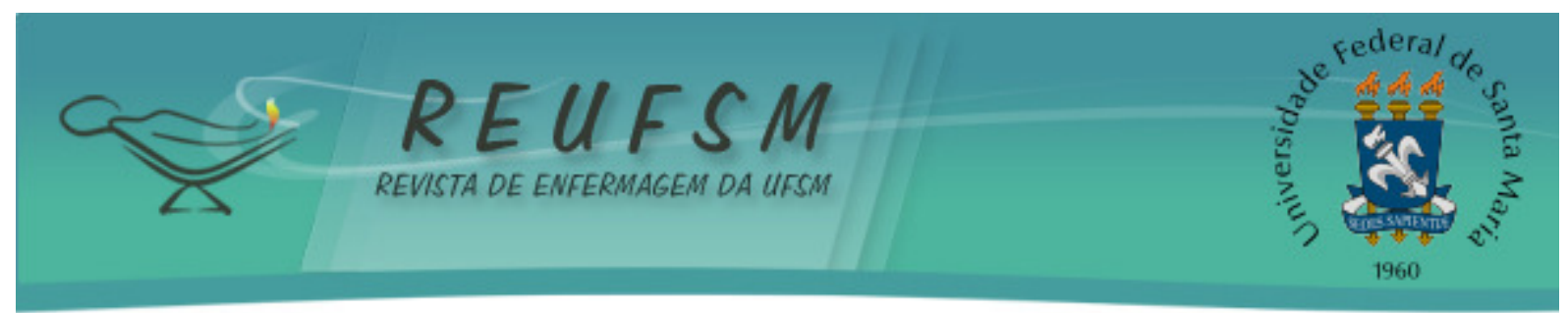

intervenções alcançaram os resultados delimitados no planejamento de Enfermagem, bem como auxiliar na mudança, aperfeiçoamento e possíveis adaptações nas etapas do PE. ${ }^{3,5}$

Entretanto, nota-se ainda que os registros de enfermagem são incompletos e insuficientes e a atuação da equipe de enfermagem se faz, por vezes, de maneira fragmentada, apresentando hiatos em seu planejamento, gerando inúmeras inconsistências na operacionalização da assistência. ${ }^{4}$ Existem, ainda, implicações sobre o custo da assistência de enfermagem, especialmente porque o planejamento e os registros das ações e resultados têm consequências diretas sobre a qualidade do cuidado, cuja análise pode viabilizar um menor tempo de internação. Há, inclusive, estudos que informam sobre a subnotificação dos registros dos cuidados de enfermagem nas contas hospitalares. ${ }^{6}$

Diante dessa realidade, entende-se que a avaliação de enfermagem e o seu registro em todos os contextos do cuidado, principalmente na UTI, são de extrema importância para a identificação das necessidades do indivíduo, para a tomada de decisões clínicas e para a execução de intervenções seguras em indivíduos com problemas multissistêmicos complexos implicando em maior segurança ao facilitar a troca de informações e permitir atenção individualizada e sistematizada. ${ }^{7}$

Tratando-se da assistência em uma unidade de grande complexidade dentro dos serviços hospitalares, as ações que o profissional executa diante da SAE devem proporcionar um atendimento qualificado e individual, promovendo a recuperação do paciente.

Desta forma, questiona-se: de que forma os enfermeiros percebem a etapa denominada avaliação no Processo de Enfermagem e a forma como se dá seu registro? Para tanto, este estudo teve como objetivo compreender as percepções de enfermeiros sobre a avaliação de enfermagem e seu registro em uma Unidade de Terapia Intensiva (UTI) adulto.

\section{MÉTODO}

Trata-se de um estudo descritivo com abordagem qualitativa, desenvolvido em uma UTI adulto de um hospital público de Santa Catarina. Na UTI em que ocorreu a coleta, como metodologia de trabalho da equipe de enfermagem, utiliza-se parte do PE com base na teoria das Necessidades Humanas Básicas (NHB) de Wanda Horta (1979), registrando-se apenas duas etapas desse processo: a coleta de dados e a avaliação. 0 instrumento utilizado para registrar a avaliação de enfermagem foi elaborado em 1995 e desde então sofreu uma única revisão, em 1999, porém, sem nenhuma alteração em estrutura e/ou conteúdo. Os registros dos enfermeiros são realizados em três turnos de trabalho (manhã, tarde e noite), abrangendo todos os sistemas orgânicos dispostos em ordem cefalopodálica.

Participaram desta pesquisa nove dos 12 enfermeiros que atuam na UTI da referida instituição. Foram utilizados como critério de inclusão: ser enfermeiro no local há pelo menos um ano. Excluíram-se deste estudo enfermeiros em férias, folgas ou licenças no período da coleta de dados. Não houve recusa de nenhum dos enfermeiros em participar do estudo.

A coleta de dados ocorreu nos meses de setembro e outubro de 2011 e adotou-se como estratégia a entrevista com roteiro semiestruturado. Para a organização dos dados da pesquisa utilizou-se o método Discurso do Sujeito Coletivo (DSC) de Lefèvre e Lefèvre. A elaboração dos DSCs segue a composição das seguintes figuras metodológicas: ExpressõesChave (ECHs), Ideia Central (ID), Ancoragem e o Discurso do Sujeito Coletivo. Neste estudo a ancoragem não foi utilizada, pois estas se referem às ideologias, valores e crenças presentes nos depoimentos, de maneira grupal ou individualizada, representados como afirmações vagas ou indeterminadas, encaixadas em circunstâncias particulares, a serem 


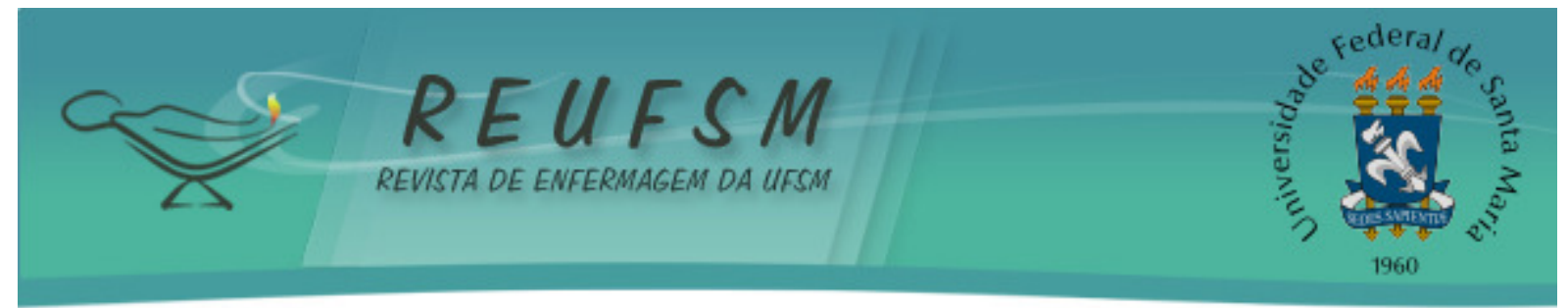

utilizadas apenas quando existem marcas explícitas das afirmações genéricas nos depoimentos, o que não ocorreu neste estudo. ${ }^{8}$

O procedimento para a construção do DSC correspondeu a seguinte lógica: leitura dos discursos individuais obtidos por meio da entrevista, agrupamento dos discursos individuais referentes de cada pergunta, identificação e destaque das ECHs de cada discurso individual por agrupamento, agrupamento de ECHs de sentido equivalente ou complementar, e nomeação das ICs. Esses agrupamentos originaram os DSC.

Para esta apresentação optou-se por não mostrar os discursos individuais e as ECHs, e sim os DSC que as contém, representados por DSC 01 e DSC 02, para diferenciá-los.

O projeto foi aprovado pelo Comitê de Ética em Pesquisa da Instituição Hospitalar parecer $N^{\circ}$ 2011/0034 e pelo Comitê de Ética em Pesquisa da Universidade Federal de Santa Catarina (UFSC) parecer $\mathrm{N}^{\circ} 2240 / 11$. Todos os participantes foram solicitados a assinar o Termo de Consentimento Livre e Esclarecido de acordo com os princípios éticos estabelecidos pela Resolução 466/12. Para preservar o anonimato, os enfermeiros foram identificados pela letra "E", seguido de número da ordem de realização das entrevistas: E1, E2, E3, E4, E5, E6, E7, E8, E9.

\section{RESULTADOS E DISCUSSÃO}

De acordo com os resultados encontrados, apresenta-se a seguir o tema e suas respectivas ICs e os DSCs, além de suas análises. Perceberam-se, então, apontamentos referentes ao que há de positivo e negativo na forma como se realizam os registros das avaliações realizadas pelos enfermeiros. Essa dicotomização gerou as categorias que seguem.

\section{Potencialidades do instrumento de registro da avaliação de enfermagem na UTI}

Ideia central: 0 instrumento de registro de avaliação é prático, rápido e serve como fonte de consulta, pesquisa e valorização profissional.

DSC 01:

Acredito que se eu avalio e dou assistência ao paciente, então eu tenho que documentar. Vejo o registro das informações como algo extremamente importante porque isso valoriza nosso trabalho, valoriza a nossa existência enquanto enfermeiro. Além disso, tem influência direta na continuidade do cuidado. Nosso instrumento de avaliação foi elaborado visando à praticidade. A característica do enfermeiro de UTI é fazer a assistência e o Processo de Enfermagem, portanto, é o nosso instrumento de adequação à necessidade de registros diários porque consegue reunir essas demandas. Além disso, são registrados dados clínicos importantes, que podem ser utilizados como fonte de pesquisa sobre a condição do paciente por todos os profissionais.

Observam-se, no DSC acima, cinco aspectos do instrumento utilizado para registrar a avaliação de enfermagem, considerados pelos entrevistados como potencialidades: praticidade, fonte de consulta acerca da condição de saúde do paciente, fonte de pesquisa e objeto de valorização profissional.

Neste contexto, a praticidade e rapidez dos registros podem ser compreendidas sob a ótica da realização de anotações ou evolução de enfermagem. Essa distinção se faz necessária, pois anotações são registros de dados brutos, pontuais, observacionais e 


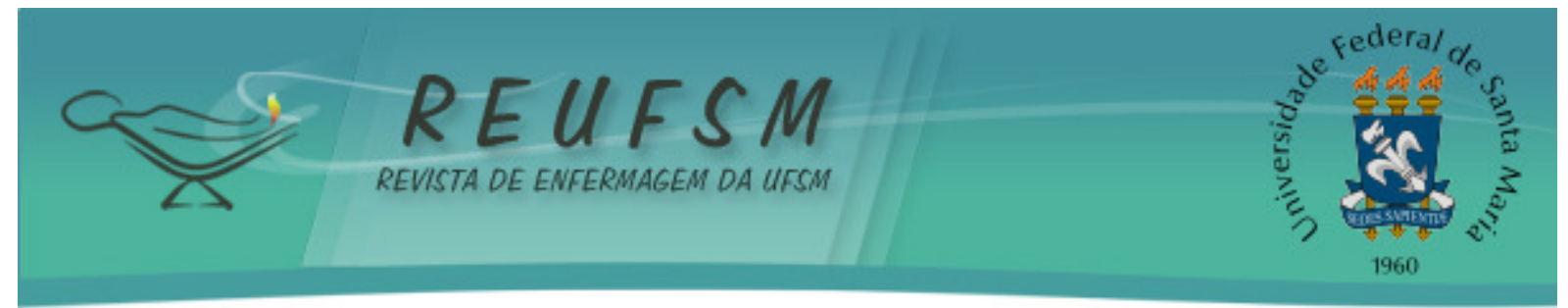

realizados por toda a equipe de Enfermagem (enfermeiros, técnicos e auxiliares de enfermagem). Já a evolução de enfermagem é a análise dos dados constantes nas anotações de enfermagem, observadas durante o período de trabalho. ${ }^{9}$

Embora a agilidade seja uma característica necessária e, por vezes, essencial em terapia intensiva, os registros de enfermagem não devem ser vistos como simples cumprimento de uma norma burocrática ou mesmo instrumento utilizado apenas para adequar-se à legislação ou normas institucionais.

Anotações isoladas, sem interpretação do estado de saúde do paciente e avaliação constante das modificações dos padrões orgânicos podem contribuir apenas parcialmente para a qualidade dos registros ou mesmo do cuidado. Apesar das anotações contidas nos prontuários acessíveis a toda a equipe na tomada de decisão, na prática, parte significativa das orientações e encaminhamentos realizados pela enfermagem não costuma ser registrada, deixando, portanto, de evidenciar a complexidade e importância do trabalho da enfermagem. ${ }^{10}$

No cotidiano assistencial da referida UTI, existem diferentes instrumentos de registros de enfermagem: instrumento de controle de sinais vitais e neurológicos, instrumento do registro do controle hidroeletrolítico, livro de ocorrências da unidade e o instrumento de avaliação de enfermagem. Essa prática fraciona as informações acarretando, por vezes, certa desarticulação e/ou desorganização dessas, ou ainda, a perda de dados. Realizar a avaliação de enfermagem de forma completa e objetiva pode configurá-la como um instrumento, a qual converge os diversos registros e informações acerca do cuidado. Porém, para tal, o amplo conhecimento acerca dos sinais e sintomas e habilidades para realização do exame físico e compreensão do estado clínico do paciente, são imprescindíveis. ${ }^{3}$

Outra particularidade do registro da avaliação dos enfermeiros é a possibilidade de se compreender medidas terapêuticas adotadas e determinar a qualidade da assistência prestada. ${ }^{11}$ Desta forma, registros rápidos e práticos podem desfavorecer a qualidade das informações se utilizados em detrimento de evoluções de enfermagem embasadas em raciocínio crítico.

Embora os participantes deste estudo tenham mencionado os registros como forma de valorização profissional, fonte de consulta acerca das condições de saúde do paciente e fonte de pesquisa multiprofissional, informações sucintas podem não contribuir para favorecer essas percepções, mas sim, fragilizar o prontuário enquanto fonte de consulta, análise e compreensão acerca das condições de saúde dos pacientes. Ao optar por um instrumento que alia a praticidade e a rapidez otimiza-se o tempo, ao passo que a qualidade dos registros pode ficar, por vezes, em segundo plano.

\section{Fragilidades do instrumento de registro da avaliação de enfermagem na UTI} consultado.

Ideia central: 0 instrumento de registro tem pouco espaço, é incompleto e pouco

DSC 02:

No nosso instrumento existe pouco espaço para o registro de informações mais amplas ou complexas, há repetição de itens e outros que necessitariam de maior detalhamento. Além disso, os aspectos sociais e psicológicos não recebem o mesmo destaque que os aspectos físicos e fisiológicos. Um fato que interfere na elaboração do registro do enfermeiro é a ausência ou má qualidade das informações complementares sobre os cuidados ao paciente e as 


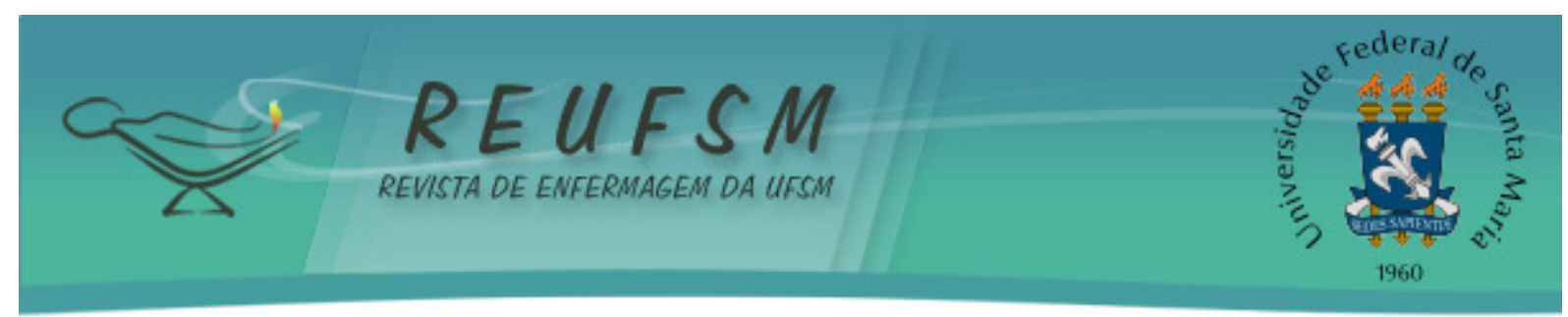

observações feitas pelos técnicos de enfermagem. Penso também que o instrumento de avaliação pode ser mais frequentemente aproveitado como fonte de consulta da situação do paciente e para isso deve ser estimulada a qualidade dos registros, para que a confiança dos demais profissionais naquilo que está escrito, possa nortear esse processo.

Conforme observado no DSC 02 , os enfermeiros identificam inúmeras fragilidades no instrumento de registro da avaliação de Enfermagem, relacionadas ao conteúdo, a estrutura da utilização do mesmo, em aparente contradição com os aspectos positivos anteriormente apontados, entrando em contradição com aquilo que apontam como potencialidades no DSC 01. A brevidade exigida pelo instrumento, que impede, pela falta de espaço, o registro de escritas mais complexas ou detalhadas da condição de saúde dos pacientes, passa a ser apontada com um ponto negativo.

Dentre as diversas interfaces que o registro da avaliação de enfermagem deve contemplar, destaca-se a necessidade da avaliação dos dados subjetivos, de cunho sociopsicoespiritual, visto que a internação em ambiente intensivo é carregada de múltiplas significações culturais que refletem na condição psíquica do indivíduo hospitalizado e de sua família. ${ }^{12}$ Essas ausências podem ser reflexos dos modelos de ensino e prática voltados, quase que exclusivamente, para os aspectos biomédicos e patológicos e cujas dificuldades ainda se fazem presentes, mesmo com o fomento de mudanças de postura e entendimento da vulnerabilidade humana nos processos de doença.

Estudo $^{13}$ apontou o despreparo dos enfermeiros por motivos que perpassam a formação acadêmica, ainda centrada no modelo tecnicista, priorizando a doença e os procedimentos, além das dificuldades em associar atividades de gerência e assistência. Há ainda fatores citados em outro estudo que aponta, dentre as dificuldades de operacionalização para sistematizar a assistência, a falta de credibilidade dos técnicos de enfermagem frente às estratégias de Sistematização da Assistência de Enfermagem, a pouca vontade dos gestores em implantá-la e o desconhecimento do funcionamento do processo de maneira irrestrita pelos enfermeiros. ${ }^{14}$

Outra fragilidade apontada nos registro da avaliação de Enfermagem é a ausência da evolução clínica de enfermagem. Na UTI em estudo, os profissionais de nível médio registram os dados referentes aos sinais vitais, características das pupilas, dados para o balanço hídrico e a presença de dispositivos invasivos (cateteres urinários, sondas gástricas e/ou enterais, cateteres venosos e arteriais, etc.), mas não há espaço para o registro das respostas do paciente observadas durante a assistência ou atividades terapêuticas propostas.

$\mathrm{O}$ PE, apesar da compreensão das razões que o tornam tão importante para a profissão, é referido como um processo bastante burocrático e de difícil operacionalização, problema que geralmente está associado ao número insuficiente de profissionais e à sobrecarga de trabalho. ${ }^{15}$

Embora os participantes não tenham feito referências à sobrecarga de trabalho especificamente, exaltaram a rapidez do preenchimento do instrumento como fator contribuinte para o processo de trabalho, fato esse que poderia ser modificado se a carga horária fosse mais bem distribuída, podendo favorecer a desburocratização da rotina dos enfermeiros. Parte dos enfermeiros utiliza com maior frequência o histórico e evolução de enfermagem, relatando não conseguir fazer o registro dessas atividades devido ao fator tempo. Estudo $^{5}$ apontou que 0 desenvolvimento do registro de enfermagem, principalmente em ambiente crítico apresentava falhas tais como: inadequações 


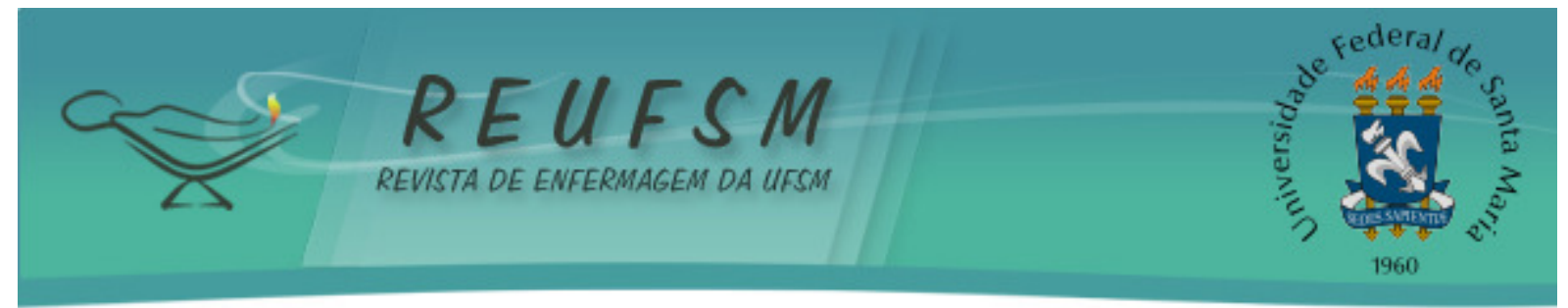

gramaticais, falta de exatidão na informação, brevidade, ilegibilidade, ausência da identificação do profissional e mau uso da terminologia técnica.

Estudo $^{4}$ documental desenvolvido a partir dos prontuários de pacientes que receberam alta da UTI de um hospital-escola do interior paranaense é citada como causa para a baixa qualidade do registro documental dos enfermeiros a má formatação dos instrumentos utilizados. As fragilidades nas construções dos formulários utilizados em terapia intensiva ocorrem, por vezes, pela duplicidade de informações, visto que isto torna a avaliação repetitiva e maçante, pois caracteriza assim uma atividade meramente obrigatória e não reflexiva, o que foi citado no DCS deste estudo.

Mesmo considerando a importância e a necessidade de documentação e registros precisos e efetivos do trabalho da enfermagem é sabido que, quanto à qualidade, o PE ainda carece de investimentos, a fim de fomentar a sua consolidação para a segurança das práticas de enfermagem. Embora seja reconhecida como atividade imprescindível, representando uma continuidade das atividades assistenciais para interpretação da terapêutica do indivíduo hospitalizado, os registros ainda são insuficientes e não efetivos nos diversos cenários de atenção em saúde no Brasil. ${ }^{16}$

A dinâmica de trabalho dentro das UTIs exige maior preocupação com a execução da assistência direta ao paciente grave, de forma intensa e constante, atribuindo erroneamente, em função disso, papel secundário aos registros ou avaliações detalhadas e embasadas em raciocínio crítico. Pesquisa ${ }^{3}$ realizada em hospital universitário apontou que há reconhecimento dos enfermeiros quanto à importância dos registros de enfermagem, mas também há descentralização e insuficiência nesses mesmos registros.

Os enfermeiros citaram ainda exiguidade de recursos humanos, falta de tempo hábil para realização dos registros e excesso de atividades administrativas e burocráticas. É preciso destacar também que a falta de tempo é citada como causa de registro incompleto, porém, é necessária reflexão dos enfermeiros acerca do desenvolvimento e priorização de atividades em serviço, visto que não é incomum que o enfermeiro assuma responsabilidades que vão além do campo de atuação, não privativas e que, possivelmente, poderiam ser delegadas a outros profissionais, em detrimento da realização das atividades prioritárias. ${ }^{3}$

A completude dos registros e sua efetiva realização têm influência direta no controle do uso de equipamentos e dispositivos invasivos, o correto uso de tecnologias, a resposta do indivíduo ao cuidado, a fundamentação de condutas futuras, o (re) planejamento da assistência, e o respaldo ético-legal. ${ }^{5}$

Diante desse contexto, há de se levar em conta que para a adequação dos instrumentos à realidade do serviço, é imprescindível o envolvimento dos enfermeiros em estudos e pesquisas sobre o tema, incentivando e buscando estratégias que contribuam para a completude dos registros. 0 cerne do problema também pode estar na organização do trabalho do enfermeiro, pois há que se destinar tempo suficiente para a realização de avaliações críticas que contribuam para o conhecimento na área e para a segurança do paciente.

A utilização do PE em todas as suas fases ainda constitui-se em enorme lacuna entre o conhecimento produzido e sua aplicabilidade no cotidiano dos enfermeiros. ${ }^{17} \mathrm{O}$ desenvolvimento do conhecimento científico na enfermagem exige formação acadêmica adequada, em que a pesquisa assuma papel de destaque e se produzam novas estratégias para fomentar a qualidade da assistência, contribuindo para as atividades inerentes à segurança do paciente. ${ }^{18}$ 


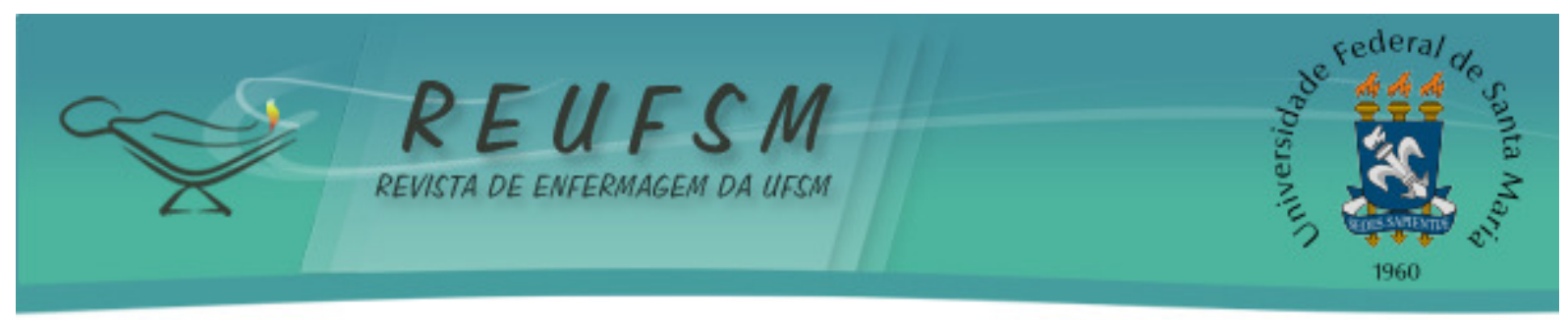

\section{CONSIDERAÇÕES FINAIS}

Considerando-se os objetivos deste estudo, os resultados apontaram como potencialidades do instrumento de registro de avaliação da Enfermagem da referida UTI: a praticidade, a rapidez para o registro, o documento enquanto fonte de consulta da condição do paciente, o documento como fonte de pesquisa e como instrumento de valorização profissional. Como fragilidades foram destacadas: a brevidade do documento com limitação de espaço para registro, critérios de avaliação insuficientes e incompletos, além da duplicidade de critérios de avaliação.

Dentre as fragilidades apontadas pelos sujeitos consta, dentre outros, o registro insatisfatório dos aspectos sociopsicoespirituais durante a avaliação de Enfermagem. Esse achado permite inferir que os enfermeiros ao valorizarem os aspectos físicos em detrimento dos psicossociais, revelam a dificuldade nessa abordagem deixando de oferecer um cuidado que contemple as várias dimensões do ser humano independente do contexto de cuidado.

Os discursos do sujeito coletivo, formulados a partir dos resultados deste estudo, mostram que o PE permanece como uma atividade que ainda carece de investimentos para compreensão e utilização irrestrita. Sua operacionalização na prática é ainda insipiente, fragmentada e não potencializa o reconhecimento sobre a importância do cuidado seguro e fortemente embasado em evidências.

Há a necessidade de estudos que investiguem sua aplicabilidade, teorização e adequação à realidade local, não apenas diagnosticando, mas também propondo, esmiuçando estratégias e propiciando novas possibilidades. No cenário deste estudo, a utilização das etapas de coleta de dados e avaliação não caracteriza a utilização do PE, mas, sim uma forma fracionada de sistematização da assistência de enfermagem.

Outro aspecto importante a ser ressaltado é o fato de o instrumento utilizado ter passado pela última revisão há 13 anos. A importância teórica dos referenciais utilizados está presente, porém, há concepções que podem ser superadas e as discussões entre os enfermeiros pode gerar instrumentos adaptados à realidade local, otimizando-os para garantir o raciocínio crítico como base das ações. O tema precisa ser discutido e as possibilidades de avanço amplamente avaliadas, propondo-se alternativas e possibilidades de modificação ou adequação dos instrumentos, de forma a contribuir com a qualidade da assistência.

A importância da qualidade dos registros acerca do que o enfermeiro avalia nos pacientes em terapia intensiva, tem sido uma característica continuamente aventada e reforçada em sua importância na formação continuada dos profissionais e, inclusive, na formação de estudantes de enfermagem. Os resultados mostraram que registros objetivos e criteriosos podem também melhorar a comunicação entre os membros da equipe de saúde. Salienta-se ainda que registros de excelência possam constituir-se em garantia de menores perdas econômicas, fortalecimento da enfermagem como ciência, além de ser um requisito válido do ponto de vista jurídico.

\section{REFERÊNCIAS}

1. Pedreira MLG. Práticas de enfermagem baseadas em evidências para promover a segurança do paciente. Acta Paul Enferm. 2009;22(Especial 70 Anos):880-1.

2. Conselho Federal de Enfermagem (COFEN). Resolução COFEN n॰ 358, de 15 de outubro de 2009. Dispõe sobre a Sistematização da Assistência de Enfermagem e a implementação do Processo de Enfermagem em ambientes, públicos ou privados, em que ocorre o cuidado 


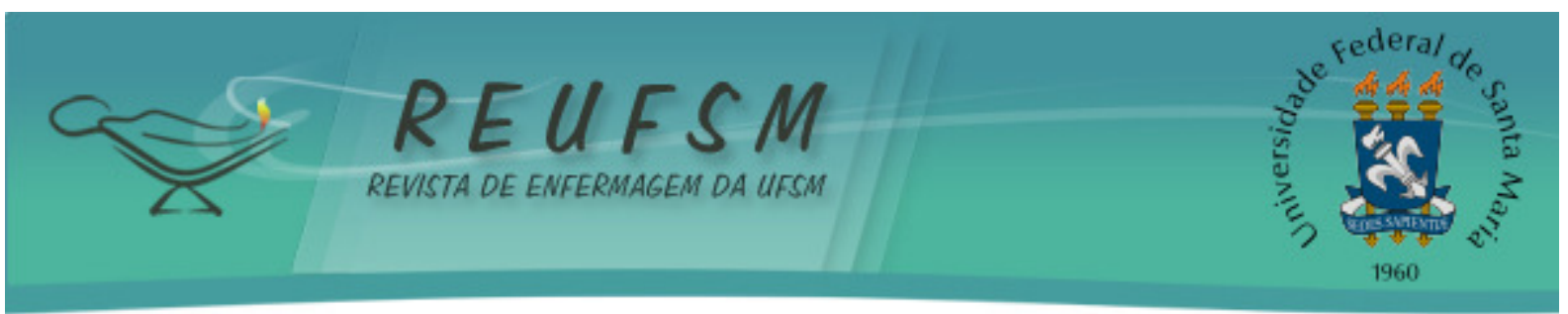

profissional de Enfermagem, e dá outras providências.. Rio de Janeiro: Conselho Federal de Enfermagem; 2009.

3. Pimpão FD, Lunardi Filho WD, Vaghetti HH, Lunardi VL. Percepção da equipe de enfermagem sobre seus registros: buscando a sistematização da assistência de enfermagem. Rev Enferm UERJ. 2010;18(3):405-10.

4. Seignemartin BA, Jesus LR, Vergílio MSTG, Silva EM. Avaliação da qualidade das anotações de enfermagem no pronto atendimento de um hospital escola. Rev RENE [Internet]. 2013 [acesso em 2014 ago 18];14(6):1123-32. Disponível em: http://www.revistarene.ufc.br/revista/index.php/revista/article/view/1352/pdf.

5. Borsato FG, Rossaneis MA, Haddad MCFL, Vannuchi MTO, Vituri DW. Avaliação da qualidade das anotações de enfermagem em um Hospital Universitário. Acta Paul Enferm. 2011;24(4):527-33.

6. Blanck CY, Sanches EN, Leopardi MT. A prática do enfermeiro auditor hospitalar na região do Vale do Itajaí. Rev Eletrônica Enferm [Internet]. 2013 [acesso em 2014 ago 18];15(1):233-42. Disponível

http://www.fen.ufg.br/revista/v15/n1/pdf/v15n1a27.pdf.

7. Lunney M. Use of critical thinking in the diagnostic process. Int J Nurs Terminol Classif. 2010;21(2):82-8.

8. Lefévre $F$, Lefévre AMC. O discurso do sujeito coletivo: um novo enfoque em pesquisa qualitativa (desdobramentos). Caxias do Sul: EDUCS; 2003.

9. Santos MGPS, Medeiros MMR, Gomes FQC, Enders BC. Percepção de enfermeiros sobre o processo de enfermagem: uma integração de estudos qualitativos. Rev RENE [Internet]. 2012 [acesso em 2014 ago 18];13(3):712-23. Disponível em: http://www.revistarene.ufc.br/revista/index.php/revista/article/viewFile/739/pdf.

10. Assunção RC, Dalri MCB. Avaliação dos aspectos éticos e legais dos registros de enfermagem. Ciênc Cuid Saúde. 2010;9(4):676-81.

11. Oliveira R, Maruyama SAT. Princípio da integralidade numa UTI pública: espaço e relações entre profissionais de saúde e usuários. Rev Eletrônica Enferm [Internet]. 2011 [acesso em 2014 jun 21];11(2):375-82. Disponível em: http://www.fen.ufg.br/revista/v11/n2/v11n2a19.htm.

12. Setz VG, D'Innocenzo M. Avaliação da qualidade dos registros de enfermagem no prontuário por meio da auditoria. Acta Paul Enferm. 2009;22(3):313-7.

13. Moura ACF, Rabelo CBM, Sampaio MRFB. Prática profissional e metodologia assistencial dos enfermeiros de um hospital filantrópico. Rev Bras Enferm. 2008;61(4):476-81.

14. Medeiros AL, Santos SR, Cabral RWL. Desvelando dificuldades operacionais na sistematização da assistência de enfermagem na perspectiva da Grounded Theory. Rev Eletrônica Enferm [Internet]. 2013 [acesso em 2014 jun 29];15(1):44-53. Disponível em: http://www.fen.ufg.br/fen_revista/v15/n1/pdf/v15n1a05.pdf.

15. Alves AR, Lopes CHAF, Jorge MSB. Significado do processo de enfermagem para enfermeiros de uma unidade de terapia intensiva: uma abordagem interacionista. Rev Esc Enferm USP. 2008;42(4):649-55. 


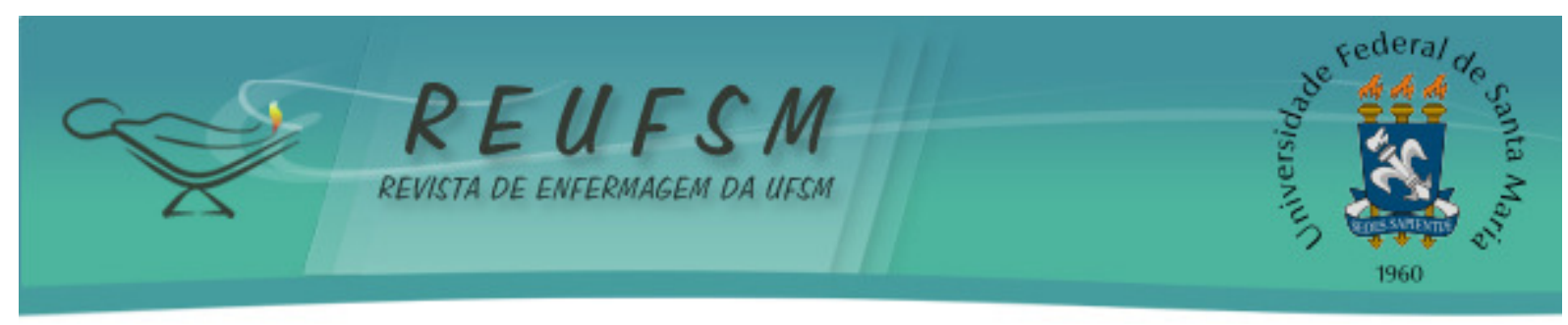

16. Maziero VG, Vannuchi MTO, Haddad MCL, Vituri DW, Tada CN. Qualidade dos registros dos controles de enfermagem em um hospital universitário. REME Rev Min Enferm. 2013;17(1):171-6.

17. Conceição VM, Araujo JS, Oliveira RAA, Zago MMF, Souza RF, Santana ME, et al. Percepções culturais de acadêmicos e enfermeiros sobre a sistematização da assistência de enfermagem. Rev Enferm UFSM [Internet]. 2014 [acesso em 2014 set 17];4(2):378-88. Disponível em: http://cascavel.ufsm.br/revistas/ojs2.2.2/index.php/reufsm/article/view/11234/pdf.

18. Ferreira MA. O clássico e o emergente: desafios da produção, da divulgação e da utilização do conhecimento da Enfermagem. Rev Bras Enferm. 2013;66(N Esp):45-50.

Data de recebimento: $12 / 09 / 2014$

Data de aceite: 12/05/2015

Contato do autor responsável: Daniele Delacanal Lazzari

Endereço postal: Universidade Federal de Santa Catarina. Centro de Ciências da Saúde. Departamento de Enfermagem. Bloco H, 40 andar, sala 414. CEP 88040-900. Florianópolis, Santa Catarina, Brasil.

E-mail: danielelazza@gmail.com 